\title{
Reconciled with complexity in research on cognitive systems
}

\author{
Joanna Rączaszek-Leonardi \\ Institute of Psychology \\ Polish Academy of Sciences \\ jraczaszek@psych.pan.pl
}

\begin{abstract}
The causes of human behavior cannot be simple. Every move we make has a nested hierarchy of causes that affect its direction, timing and form. The billiard-ball type of causality that is usually assumed to explain human action cannot give sufficient justice to this complexity. In this paper, I point to those perspectives that respect the complexity of cognitive systems and recognize that cognition involves changes on many nested time scales and in many nested systems. A brief overview of methods that are suitable for dealing with such interaction-dominant complex systems is presented and used as a background for describing a specific research program with the aim of clarifying the role of language as one of the nested factors shaping cognition. I illustrate this endeavor with two studies: one concerning the development of language as interaction control and another detailing how language may shape cognitive processes on several timescales. Reconciliation with complexity leads us to ask slightly different questions and expect different answers than when using simplified componential models of cognition and helps demarcate the limits of predictability.
\end{abstract}

Keywords: social sciences methodology; complexity; dynamical systems; inter-activity; language

\section{Cognitive systems and cognitive timescales}

Scientists are real biological organisms and, as such-apart from the elaborate conscious tasks they undertake as part of their day-to-day business-undergo unconscious calibration of their senses with respect to the domain under study. Extensive experience with scientific problems and methods tunes in the senses and ways of reasoning, thus allowing scientists to pick up various novel aspects of them and detect unexpected configurations. Awareness of one's own flux, or changeability, raises the need to ask oneself, from time to time, 
very basic questions concerning one's own scientific domain: for example, what is the object of study, or what are the main goals. For a cognitive scientist, these should be questions about the basic nature of cognition and the main motivations behind studying it. Recently, while revisiting the main assumptions of my research endeavors, I decided to ask around at major cognitive science conferences and smaller meetings the question, "What, for you, is cognition?" Where does it start, evolutionarily? What are its defining features? On which timescale do cognitive processes happen?

I was surprised by the diversity of answers I received: some of them were brief, and these were roughly "cognition is information processing by the brain/nervous system", reflecting the dominant approach to cognition for the last few decades. However even within this group, the answers to other questions, e.g., those regarding the evolutionary complexity at which cognition appears, were not as uniform, placing it on a continuum ranging from organisms with relatively simple nervous systems, such as C.Elegans, through insects, mammals, to even only in humans. Others, however, were much more liberal, ascribing cognitive abilities to bacteria and their colonies, or plants. The criteria for cognition ranged from an ability to anticipate and plan, often related to constructing internal models of the outside world, to more "permissive" answers, which classified as cognitive all living systems able to self-maintain and reproduce, in a way treating evolution as cognition on a slow timescale.

As to the timescales of cognitive processes, the most frequent answer was that cognition involves the ability to use and transform information here and now in order to guide the present and anticipate future behavior. Since many researchers have underscored an unchanging organism's ability to transform information, rather than transformations of organisms according to information, this meant practically excluding evolutionary processes as being cognitive and treating developmental (and even learning!) processes as ways of arriving at a proper cognitive system rather than cognition per se. Others, again, were also much more inclusive in the matter of timescales for cognition, indicating that forming an organism on timescales longer than the current one also seems to "solve" particular environmental challenges and enables self-sustaining and reproduction. This inclusive stance also makes less obvious the usual choice of an individual organism as the only site of cognitive processes. The units within which problems of adaptation and selfmaintenance are solved may include both sub- and supra-individual levels. Is the immune system a cognitive system? What about a colony of bacteria? An anthill? How about a collaborating dyad? A human and a computer? A human and the Internet? A family? A group? A society? Cognition in its most general sense belongs to all those levels and timescales. 
In sum, the most crude (admittedly simplified) clustering of stances would thus include a more restrictive view of cognition as information processing by an individual organism, and a more inclusive, von Neumann-like view that emphasizes the capacity of adaptive change in complexity in the face of challenges from the environment. Taking advantage of the slightly less formal character of this piece, I have only mentioned the array of approaches as stances represented in the field, omitting the philosophical and methodological backgrounds behind those choices and their classifications into particular schools of thought (which many readers probably recognize), such as the information processing approach, ecological psychology, enactive, and embodied, extended and distributed approaches to cognition. Similarly, I allow myself to only reveal my stance rather than argument it properly (for this see e.g., Rączaszek-Leonardi, 2014; see also Rączaszek-Leonardi \& Kelso, 2008; Pattee \& Rączaszek-Leonardi, 2012). Not seeing an obvious way to separate the timescales and systems and being convinced that it is best to start with as few and as unrestrictive assumptions as possible, I feel it is safer to take the most inclusive view on cognition, preferring the danger of drowning in a multitude of systems and timescales to the danger of missing important influences and factors.

However, taking this inclusive stance comes with a serious responsibility. If cognition involves many types of changes and many nested systems from subindividual to individual to supra-individual, and happens on many nested timescales from milliseconds of neural processes to behavioral, developmental, social, cultural and evolutionary, the question is how to deal with such complexity? How to study a chosen phenomenon knowing that it is always intertwined in such a hierarchy of influences?

\section{Approaches to complexity}

Treating cognition as a set of rather clearly delimitated processes that happen in the current timescale in an individual mind/brain often comes with the comfortable assumption that the system under study can be bracketed-out from external influences and, sometimes, with an irreverent attitude towards complexity. Even though it is certainly recognized that cognitive processes are multi-componential, the components are thought to be identifiable in principle. Recent movements within this broad paradigm involve embodying and distributing cognition, subordinating it to action and "naturalizing" $\mathrm{it}^{36}$. Ho-

\footnotetext{
${ }^{36}$ This recent tendency to naturalize cognition shows that cognition became somehow denaturalized in the first place, perhaps at the moment of great bifurcation in the mid 50s when symbolprocessing machines became the leading metaphor for cognition. Naturalizing cognition understood as information processing somehow resembles trying to naturalize an artifact; for example, to explain how one could construct a bicycle having at their disposal tendons, muscles and bones.
} 
wever, in many such attempts the body is simulated within the mind/brain, i.e. it is a part of an internal model and thus reducible to more (mostly internal) components. This is a very specific understanding of embodiment and very different from, e.g., ecological and enactive approaches.

The more inclusive approaches to cognition do not have this comfort of easy bracketing. Cognitive processes are systemic in their nature, involving an active body situated in its environment. In their 2003 paper "The selforganization of cognitive performance", Van Orden, Holden and Turvey analyze the difference between the component-dominant and interaction-dominant approaches to cognitive processes. The component-dominant approach hinges on the identifiability-in-principle of the components and their functions (or sub-functions within a general function). Complexity can be tamed (e.g. by experimental design) and the explanation is reduced to effects and interactions of such identifiable components. The effects of the components, like in Donders' mental chronometry, can be discerned in the measured data. Variability, e.g., in reaction times, should consist of additive effects of the multiple components plus noise, which usually is assumed to be an uncorrelated white noise. Such additivity, however, is just an assumption. "Either additivity so far is too shy to show itself and remains to be teased out of data or it is simply the wrong assumption" (Van Orden, Holden, \& Turvey, 2003, p. 337). In contrast, it might well be-and is quite probable, given the flexibility and equifinality of cognitive function - that the moment that a component is added to a task, the whole task may reconfigure, influencing the effects of other components. In this way each component will affect all other components and the behavior will be a result of emergence from multiplicative and not additive interaction of components' effects. Further, the effects of various components may unfold over various timescales, leading to non-stationarity of the measures. The timescale of an experiment is nested in many other timescales (RaczaszekLeonardi, 2010; Wallot \& Van Orden, 2011b).

Recent years bring an increasing body of evidence that the latter view better reflects the nature of cognitive systems than the component-dominant view. Research demonstrates variation to be correlated across time, taking the shape of "a nested structure of positive correlations" (van Orden et al., 2003, p. 335). Van Orden's own research group has shown this in many studies, including reading times data reported in the 2003 paper. Others reveal this in multiple domains of cognition, including "controlled cognitive performances [such as] mental rotation, lexical decision, visual search, repeated production of a spatial interval, repeated judgments of an elapsed time, and simple classifications (Aks, Zelinsky, \& Sprott, 2002; Clayton \& Frey, 1997; Gilden, 1997; Gilden et al., 1995; Kelly, Heathcote, Heath, \& Longstaff, 2001)." (Van Orden et al., 2003, p. 343). Moreover, measures that many researchers depend on were found to be non-stationary, showing the presence of influences from many nested timescales. For example, Wallot, Hollis \& van Rooji, 2013, demon- 
strated non-stationarity for reading time, a habitually used measure in language comprehension that seems to decrease indefinitely with the amount of text being read.

These findings threaten the very bases on which the methodology of most current research rests: i.e., the assumptions of the uncorrelated noise, which should take form of random white noise on the background of which the components' effect could be measured, or the assumption that measures we take reflect only the effects of independent variables (plus noise) and are stationary. From time to time, the non-stationarity of measures is discussed in cognitive research and the assumption of stationarity is criticized (see e.g., Deary, Caryl, \& Gibson, 1993), yet no systematic approach to the problem seems to emerge. With the basic assumptions threatened and the possibility that the non-additivity, nonlinearity and reciprocality of the influences makes the components' effects inextricable from the effect of other components, it seems that other research strategies have to be sought to augment the construction of "subtraction" situations and designing simple (or even more complex, e.g. structural) additive models.

According to the interaction-dominant approach, everything that can be measured is an effect of complex interaction, thus the interaction itself and its properties become the main object of study. Instead of asking what the components exactly are, or how to decompose a cognitive function, we rather ask: how complex is the system realizing a function, is it more complex in one situation than in another, what kinds of interactions seem to be at work? Behavior in experimental conditions is thus not an effect of recruiting (or dropping) another component from an otherwise unchanged cognitive system, but rather a reorganization of the system under novel task constraints.

In interaction-dominant systems it is assumed that each and every behavior is nested in a hierarchy of constraints, originating in a hierarchy of systems nested in a hierarchy of timescales. Van Orden gives an example of catching soap while showering: the effectiveness and the trajectory of our hand catching it will reflect this nested goals' structure. As Merleau-Ponty put it, it will reflect the hierarchy of projects we are committed to (Merleau-Ponty, 1962). They include saving the soap, taking a shower, being clean and not hurting oneself in the act of catching. Taking a more cognitive example, consider a situation in which an aesthetic judgment is made. If one asks what it is that makes a person choose one work of art over another, a multitude of influences from multiple systems and timescales may be in play. The systems involved would include an individual, the ambience she grew in, the family, school, social group, culture and even the species. The timescales on which those preferences are shaped include the present situation, the contrast with particular works of art seen immediately before, the mood, the sharpening of artistic sensibility in education, experience, the aesthetic evolution of a parti- 
cular culture and the properties of a perceptual system tuned to particular properties in biological evolution.

Reconciliation with complexity makes us thus turn to synthetic models, giving up applying the physics-motivated analytical ones as unsuitable for living organisms in most cases (e.g., Rosen, 1991). The situation is complex, but much work has already been done to show how to deal with such complexity. It shows that even without identifying functional components we can study complex systems and be able to predict and change their behavior. We can discover and not assume functions: we do have ways to relate them to values realized in the environment, we do have methods for dealing with nested structures of nonlinearly interacting components over many levels and timescales. We can learn a lot about a system: instead of reducing it to several variables, we can try to reconstruct the dynamics of the entire system from the chosen variables. Finally, we can discover points of leverage to change a system even without knowing its detailed componential mechanics. Alternatively, we can more clearly see where complexity leads to unpredictability and a lack of control. This has advantages as well: Firstly, for a human scientist it must be a kind of relief that there are limits to the predictability of human behavior. Secondly, it is better to admit less control than to be patently wrong.

\section{Respecting complexity: How to deal with interaction-dominant systems?}

How to study a system about which we know that: 1) its components are unknown and possibly unidentifiable, 2) measures that we can take from its behavior might be non-stationary and 3) the variance of those measures cannot be easily partitioned.

One approach that is at ease with complexity and the violation of uncorrelated noise and stationarity assumptions is the dynamical systems approach. It requires changing our models, changing the types of research questions and using particular measures and methods, which take well (are designed to deal with) non-stationarity, non-linearity and non-independence of error sources. This approach takes the weakness (that everything influences everything else) as its strength: that therefore every variable reflects something of the whole system.

This short paper is not a place to review the now very extensive literature on the application of methods to study complex systems in behavioral and social sciences (Guastello \& Gregson, 2016; Jeka \& Kelso, 1989; Kelso, 1995; Schöner \& Kelso, 1988; Thelen, 1992; Thelen \& Smith, 1996), Port \& van Gelder, 1995; Guastello et al., 2009). In the following paragraphs I try to give several examples of concrete research to illustrate the kinds of questions asked, the measures which operationalize key concepts, the methods used to analyze these measures and the types of conclusions being drawn from them. It will become clear 
that instead of the usual cognitive psychological measures such as reaction time, accuracy of performance and activation, all of which are chosen to characterize outcomes and bases of actions, the emphasis will be on actions themselves: the form of trajectories, variability, stability, compensatory processes, reaction to perturbation, dimensionality, coupling strength, etc. Often, instead of searching for an assumed function, the research process starts with detecting functional stabilities (synergies, stable configurations of parts) and investigating parameter-dependent changes.

One of the classic works exemplifying identification of synergies is Kelso et al.'s 1984 paper, in which they studied compensation of perturbations in syllable production. A lower jaw prosthesis was attached to the torque motor in a way that allowed it to deliver additional unexpected loads to the jaw during its upward motion in syllable production. The activity in the upper and lower lip and tongue muscles was measured and reactions to perturbations were assessed. Immediately after a jaw perturbation, a compensatory movement was observed in a syllable-specific effector: this movement was strongest for both the upper and lower lip when the syllable was /beab/ and was strongest in the tongue when the syllable was /baez/. The delay with which these compensatory movements occurred was extremely short (20-30ms); however, this could not be attributed to some reflexive unspecific coupling of effectors because the compensation appeared only when functionally sensible, e.g., when the perturbation was delivered to the upward moving jaw and not when it was delivered during the downward motion (Kelso et al., 1984). This work shows that syllable production can be characterized as a formation of functional synergy, for which all parts of the vocal tract are coordinated for a specific goal.

Once such synergies are identified (for example, by the properties of reaction to perturbation, persistence under parameter change, flexible organization, equifinality), their stabilizing and destabilization conditions can be studied, which (among others) allows for finding "handles" for their control. Gait changes with increasing speed were one of the first phenomena under study; however, an important advancement in description and modeling of such phenomena was made when bimanual coordination in humans was studied in Scott Kelso's lab (see, e.g., Kelso, 1995). In this setup, participants were asked to flex their index fingers in or out of sync, paced by a metronome. In the out-of-sync condition, the increase in frequency results in a rapid transition into an in-sync mode. Signatures of self-organizing synergies were experimentally demonstrated in this situation, as bistability of the system at low frequencies gave way to monostability at a certain critical frequency. Finding the right variable to characterize the global stable states of a system (order parameter) and variables which are able to bring the system from one stable state to another (control parameters) was crucial. In this case, the order parameter was the relative phase of the moving fingers, and the control parame- 
ter was the frequency of movement. A model which captures the complexity of this behavior is a dynamic equation of motion, in which-depending on the value of the control parameter-the system has two stable states (at 0 and 180 degrees) or only one (at 0 degrees) (Haken, Kelso, \& Bunz, 1985). Switching from one state to the other occurs due to a loss of stability, which is captured by the model. Observable patterns of behavior, such as hysteresis (i.e., holding on to an already stable behavior as the control parameter varies continuously) and variability patterns are successfully modeled by the HKB model (the latter by its stochastic version).

Similar models were designed not only to capture a variety of other movement organizations-demonstrating their self-organized, synergetic character-but also to model the phenomenal stability of perception. Models for switching of the Necker cube, perception of apparent motion (Hock et al., 2003), consonant and syllable perception (Tuller, Case, Ding, \& Kelso, 1994) and even sentence perception (Rączaszek, Tuller, Shapiro, Case, \& Kelso, 1999) were also designed and their predictions experimentally tested. The models were also suitable to integrate slower timescales and have been used to model learning movement synchronizations or learning new perceptual contrasts, such as the emergence of new stable states (Zanone \& Kelso, 1997; Case \& Tuller, 1995). Based on brain imaging data, similar models have been designed to predict brain activity during such motor or perceptual tasks (Jirsa, Fuchs, \& Kelso, 1998).

Questions about the quality of coordination and strength of coupling of the elements of a system can also be answered by studying the resulting system's dimensionality, which is a measure of how well a system reduces its overall degrees of freedom to perform a specific task. One can ask such question on many levels: the individual, as in movement coordination, but also the interindividual, on which people coordinate to achieve a common goal. Riley et al. (2011) showed that this method can be informative in the study of interpersonal synergies. Using principal component analysis, they investigated the number of dimensions that account for variance in the trajectories of 12 joints in a task requiring coordinated movement. The more correlated the variables of the system, the more they are influenced by each other and the higher the dimensional compression (thus lower dimensionality). Riley et al. observed reduction of dimensionality in a cooperating dyad greater than in an individual person, which reflected the constraining influence that persons exerted on each other within the task.

A similar goal (i.e., assessing the strength of interpersonal vs intrapersonal coordination) can be achieved by another method. Uncontrolled Manifold (UCM) analysis (Scholz \& Schöner, 1999) can be thought as a measure of the functional coupling of a system, roughly as a measure of reciprocal compensation. The UCM specifies a sort of "corridor" in the state space of a system, in 
which the variability of a movement will be compensated for in such a way that the function is still properly performed. Romero et al., (2015) used this method to show that jointly performed actions were synergistically organized both at the intrapersonal and interpersonal level, with the interpersonal synergy significantly stronger than the intrapersonal synergies. This was an elegant demonstration that one can think about a dyad coordinating in a task as a novel system that self-organizes its dynamics under task constraints (Romero, Kallen, Riley, \& Richardson, 2015).

Another very useful method that has recently been very popular among dynamical systems researchers is recurrence quantification analysis (RQA). This method provides an excellent example of the research philosophy proposed by van Orden et al., 2003: reconstruction of the dynamics of a system from a single or several variables measured in time. The propensity of a system to recur in the same neighborhoods of its state space and the time spent there testifies to the system's complexity and identifies regions of stability. It can also be used (cross recurrence analysis or cRQA) to quantify the relative strength and the nature (e.g. entraining direction) of coupling between two systems. The method is useful for studying interactive dynamics as will be demonstrated in an example below.

In sum, dynamical systems methods show that a lot can be achieved without assuming a componential, simplified model: identification of functional stabilities of coupling with the environment, studying stability and destabilization under certain parameter values and studying the compensatory structures of synergies. Mathematical models designed to capture the main properties make it possible to predict the behavior of the system given specific parameter values and the system's history. Initially restricted to movement sciences, this approach is presently being developed to encompass phenomena considered as more "cognitive", such as perception or problem solving (e.g., Stephen et al, 2009).

\section{Interaction-dominant systems in the domain of language}

In the assumed reality in which everything is interaction, there seems to be little space for the most important explanatory concept in cognitive science: that of symbols, which seem indispensable when talking about such important aspects of cognition as thinking and language (if not about cognition in general) (for a more detailed discussion of this point see Pattee \& RączaszekLeonardi, 2012; Rączaszek-Leonardi, 2014). The dynamical systems approach and its methods did, however, also reach into the domain of language: examples include the aforementioned study of reading times (Wallot, Hollis, \& Rooij, 2013; Wallot \& Van Orden, 2011a, 2011b), using recurrence analysis to show the properties of dynamics that underlie certain text structures (e.g., 
Orsucci et al, 2006) or even creating dynamical models of parsing (beim Graben et al., 2008).

However, despite this research, only limited attention has been given to the very nature of symbolic entities and to the connections (forms of causal relations) that they might have to the dynamics in which they are immersed. Therefore, in our work (Rączaszek-Leonardi \& Kelso, 2008) we are seeking to clarify this nature, the ontological status of symbols and their structures, as well as the functional relation they have to the dynamics within which they arise. This approach has a broader scope (Pattee \& Rączaszek-Leonardi, 2012), but focuses mostly on one symbolic system: natural language (RączaszekLeonardi, 2014, 2016). The main goal is to show the role of language in human interactive dynamics and to explain how it is possible that, amidst all these dynamics, language acquires syntactic and formal properties. In a theoretical framework we have developed over the years, taking a more cybernetic than purely cognitive view, we propose that language, rather than describing or referring to the world by some form of mapping, functions as a system of controls over human purposeful interactive dynamics. Communication thus, rather than as a "meaning transfer", is seen as a formation of collective systems in a process of mutual constraining. Structures of language are replicable constraints which arise in a rich history within the systems they control over cultural time scales (Rączaszek-Leonardi, 2012).

According to this approach, the meaning of linguistic forms cannot be reduced to some kind of simple association to a mental concept (or a category of objects), but rather has to be retrieved from the role of these forms in constraining certain degrees of freedom of an interactive system. Thus the relation between symbols and dynamics is more complex than a simple association, but also less mysterious than, e.g., "mental interpretation". Through cultural evolution, structures of language become effective controls on (already existing) interactive dynamics. Including dynamical action and interaction as vital elements in the specification of linguistic meaning alleviates the problems of contextual dependency of the meaning or efficiency of language (see Rączaszek-Leonardi \& Kelso, 2008; Rączaszek-Leonardi, 2016), but puts a heavy burden on the shoulders of a scientist in that she is expected to identify the relevant dynamics and specify how control through language may be realized. Our research is devoted to this and below we show examples of two studies and a sample of the theoretical work in which we are engaged for this purpose.

\section{Recognizing purposeful dynamics of parent-infant interaction}

The study of the ground - the natural habitat-in which language develops as a specific control of human coaction was the motivation behind our microanalyses of parent-infant coordination (Rączaszek-Leonardi, Nomikou, \& Rohl- 
fing, 2013; Rączaszek-Leonardi \& Nomikou, 2015; Nomikou et al., 2016, Leonardi et al., 2016, Rączaszek-Leonardi, 2016). We sought to uncover the properties of the interaction dynamics in order to be able to study how language shapes it further. We describe (Rączaszek-Leonardi et al., 2013) how, already from the $3^{\text {rd }}$ month of life, mothers encompass infants' actions within their own, thus recreating the interactive routines present in a given culture. We have pointed to the participation in such routines as a factor which educates the attention of an infant and tunes his perception to movements of others as possible social affordances, as well as the infant's own movements as affordances to others. Bodily actions become meaningful in this way by having a place in a larger, purposeful routine. After Heft (Heft, 1989), we have shown that the behavior of a child might become intentional by immersion in a larger coactive structure and not necessarily through a mediation of an elaborate theory of mind in which intentions of others are represented. We have illustrated how mothers go out of their way in recreating such meaningful events around infants' initially random actions (Rączaszek-Leonardi et al., 2013). We also underscored and illustrated with real life examples how such "movement-first" and "form-first" instilling of patterns of states and behaviors is saturated with cultural values, not just directed to immediate goals (Rączaszek-Leonardi \& Nomikou, 2016).

This work was mainly theoretical, albeit illustrated by qualitative microanalyses of videotaped natural interactions (for corpus description see Rohlfing \& Szufnarowska, 2011). Subsequently, we also employed dynamical time series analyses (the aforementioned RQA) to show the progressive structuring of the interactions. For example, we analyzed gaze-at-face behaviors of mothers and infants. Figures $1 \mathrm{a}$ and $1 \mathrm{~b}$ show the average recurrence profile of motherinfant gaze behavior, with the line at point 0 corresponding to the frequency of displaying the same behavior by mother and infant at the same time (lag 0 ), while the left side of the graph corresponds to mother following the child's behavior and the right side to infant following the mother. As we can see, there is no clear leader and follower in gaze behavior and they become more tightly coupled (dependent on each other) with age (peaking at 0 lag becomes more pronounced) (Nomikou et al, 2016). Following more carefully the age progression of this process, we observed that the pattern of gaze that is typical of interactions with 3-month-old infants, in which they and their mothers gaze a lot at each other (see Figure 1a), gives way to patterns of less and shorter gazing, albeit much more tightly coupled in time, which is especially evident after normalization (Figure 1b). 


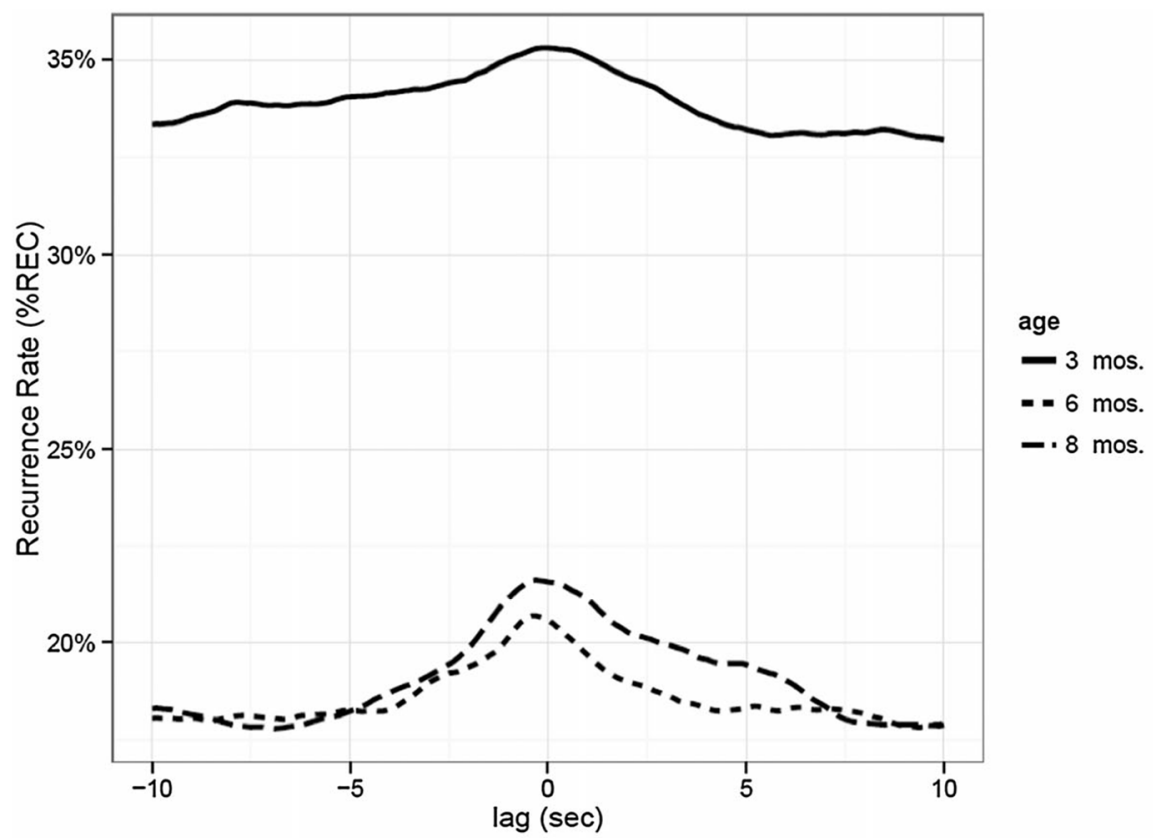

a

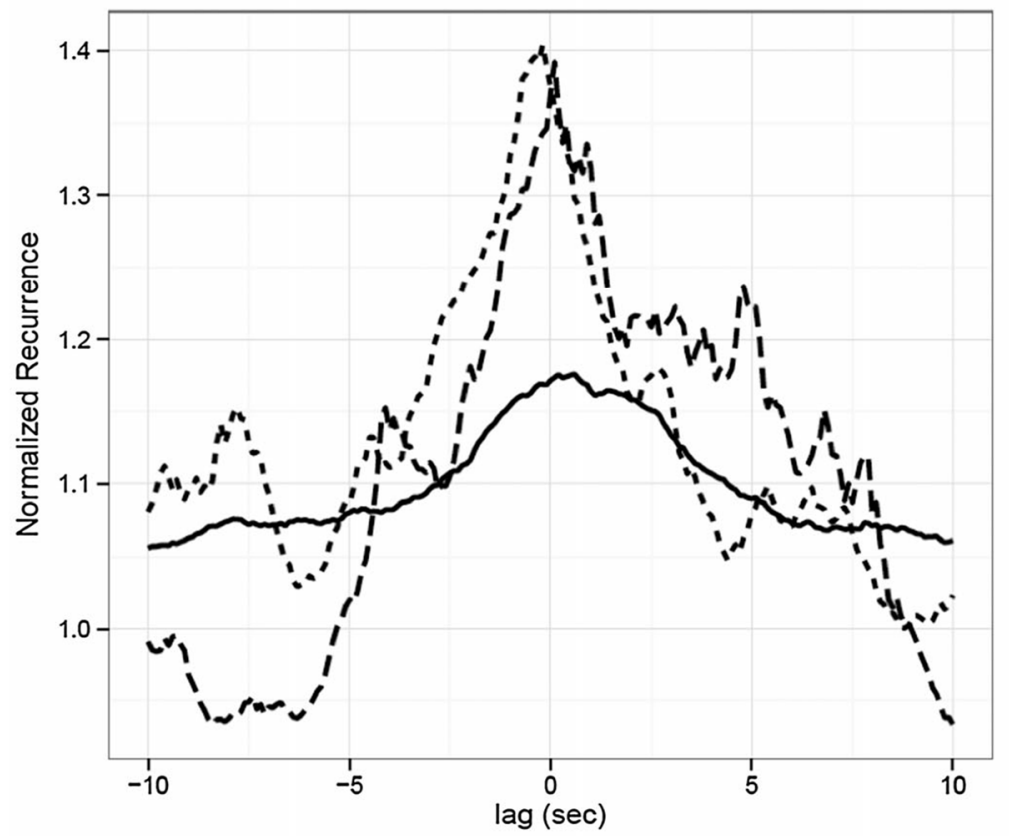

age

-3 mos.

-6 mos.

$-.8 \mathrm{mos}$.

Figure 1: Cross-recurrence lag profiles (roughly, probability profiles of matching behaviors as a function of time lag between participants) for gaze-at-face behavior. 1a shows cross-recurrence lag profile before normalization, which illustrates how much more frequent mutual gaze-at-face is at 3 months of age. Normalization (1b) allows to one see how the coupling of gaze becomes tighter (higher peak at 0 lag) at 6 and 8 months compared to 3 months of age. Figure reprinted (with permission*) from Nomikou et al., 2016. 
Looking at coupling in a different modality, namely vocalizations (Figure 2), we can see that mothers closely followed the vocalizations of the child at 3 months of age (a peak to the left of the lag 0 line), which then gives way to stabilizing the turn-taking structure, i.e., avoiding vocalizations at the same time (evident as deepening of the valley at 0 lag, just the opposite of the peaking structure of the lag profile in the case of gaze) (Leonardi et al., 2016). Our further plans include pursuing such dynamical analyses in multiple modalities, which should give us a fuller picture of the emergent interactive routines. It is these routines that are further shaped by language, which, at early developmental points, is probably no more informative than any other action or gesture.

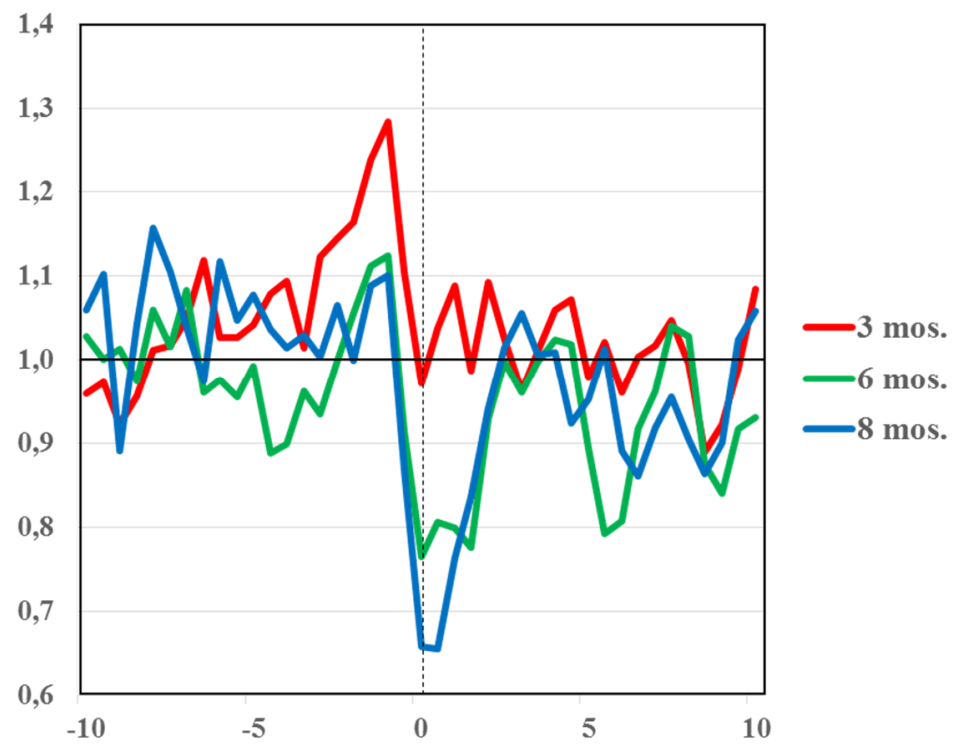

Figure 2: A cross-recurrence profile of vocalizations, showing the emergence of turn-taking structure at 6 and 8 months.

\section{Language as a control on the purposeful dynamic of parent-infant inte- raction}

The continuation of this project involves investigation of how language further transforms such interactive patterns. This involves theoretical work on the kinds of relations linguistic utterances might have with respect to the dynamical events unfolding within a situated interaction as well as empirical work, both exploratory and aimed at verification of this theoretical framework.

To achieve this goal we have employed Peirce's semiotic distinctions, on the basis of which Deacon developed his views on how the emergence of symbolic systems depends on other semiotic processes (Deacon, 1997). Applying this 
view to language development, we are working on an approach to language acquisition in which the involvement of symbol vehicles in indexical and iconic relationships on two levels is crucial: one is the pragmatic, constraining relation to ongoing events, within the interactive dynamics as illustrated above; the other is the indexical and iconic relation among the symbolic vehicles, sustained by (and comprising) a language. We propose that it is the relation between these more basic forms of signification that constitutes a developmental transition to a truly symbolic system (Rączaszek-Leonardi \& Deacon, in preparation).

This theoretical work is complemented by empirical research. The exploratory part involves an investigation into differences in the multimodal dynamical surroundings of infant's language-like and non-language-like vocalizations. We are curious about the methods parents use to make the first utterances of the child significant by letting them have specific social consequences. Complementarily, we investigate the "tricks" that parents use to make infants' behaviors "appropriate" to the utterances of a parent. A good example is a parent who calls a child's name and then, if the child does not turn to meet the parent's gaze, touches the child or tickles her and, if this still does not work, looms over the child or in some other way tries to meet her gaze. The importance seems to be given to preserving specific patterns of actions: calling a name, a sustained look, a smile. Differences in treatment of language-like and non-language-like vocalizations are differences in sequencing and timing of actions; thus, methods of time series analysis, including dynamical ones (such as Markov chains, assessment of entropy, rqa and crqa) are suitable for studying them. The empirical attempts at verifying this dynamico-semiotic model of language acquisition are in the early stages. They involve, at this point, the identification and qualitative description of the processes that the theory proposes are at work. Initially, at an early age, symbol vehicles begin to function as indices and icons in social interaction (including the processes mentioned above); subsequently relating them (via indexical and iconic relationships) to other symbolic structures liberates them from the immediate context (Rączaszek-Leonardi et al., in preparation). The focus is on finding particular cases of words and trajectories in their interactive history that would illustrate this process and, subsequently, on supporting the hypotheses with suitable dynamic and statistical analyses. This should result in a better understanding of how language remains grounded in interactive dynamics, which preserves its controlling powers, while at the same time gains the properties of a symbolic system, with its conventionality and formal properties (Rączaszek-Leonardi \& Deacon, in preparation). Such an approach gives a glimpse on how complex the characterization of linguistic meaning is, but also hints at the possibility of connecting the dependence of meaning on bodily dynamics to its reliance on systemic relations. 


\section{Performance of collective systems controlled by language}

Developmental research is a good domain for tracing the emergence of symbolicity and investigating language's dependence on (and the emergent independence of) interactive bodily dynamics. However, the dynamical systems approach can also lead to novel testable hypotheses in the area of human taskoriented communication. With the view of language as a control system over interaction-dominant collective systems, researchers can focus on how reliable controls emerge in task situations and what kind of organization and reorganization of collective systems they bring, which in turn helps predict the results and variability of task performance.

Recent research (Fusaroli et al., 2012; Fusaroli et al, 2016) has demonstrated that the effectiveness of communication in task situations cannot be reduced to pure linguistic alignment, which by most models is considered the primary index of how much people understand each other (Pickering and Garrod, 2004, but see Healey, Purver \& Howes, 2014). Indiscriminative alignment is not predictive of better performance by dyads in perceptual tasks; on the contrary, it seems to be correlated with poor performance. What was more predictive was the selective alignment on those vocabulary terms which pertained to dimensions crucial for the task. In terms of our framework, the aim of this research is to establish how, in communicative situations, collective systems arrive at efficient, task-specific controls, using extant symbolic systems.

We are developing these investigations further, involving more complex and natural situations of coaction and, most importantly, searching for language influences not only on the timescale of on-line, current coordination, but also on the cultural time scale. In our recent research (Zubek et al., 2016), we sought to evidence how collective performance depends on the influence of language coordination on-line and the influence of linguistic artifacts constructed over a cultural timescale in order to deal with a particular domain. We studied individuals and pairs who performed a task of wine recognition. Half the individuals and half the pairs additionally could use a sommelier card (i.e., a cultural tool containing specific terms for wine description). Thus, we had four experimental conditions, corresponding to four kinds of cognitive systems: individuals, individuals using a cultural artifact (i.e., being influenced by the vocabulary of the sommelier card), pairs (who could spontaneously converse) and pairs using the sommelier card. In the first phase of the experiment, the participants sampled three wines in black numbered glasses with the instruction to remember them as they would have to recognize them in a larger set of wines later. Pairs could freely talk at this stage. In the sommelier card condition, one card per wine had to be filled in the learning phase; in the pair condition, participants had to negotiate the description. After a 40 minute break, participants were presented with six wines and asked to recognize which of them they had tasted before and identify them by number- 
ing them as in the original sample. Pairs, again, could talk, and participants who filled the sommelier cards could use the descriptions created in the learning phase to recognize the wines. Each pair had to agree on a common solution.

Hypotheses were posed with respect to three measures: the overall quality of performance (which we predicted would be better in pairs and better with the sommelier card), the quality of descriptions created using the sommelier card (higher for pairs, because they integrate their knowledge) and the types of error that each of the four systems would generate. The latter hypothesis, based on the above theoretical framework which treats language as a set of constraints functionally binding degrees of freedom in a given system, stated that the more linguistic controls are applied within a system, the smaller the variance of the results; i.e., in the condition with spontaneous conversation, we should have less variance in the errors committed than in the individual condition, and similarly in the conditions where the sommelier card was applied as an additional constraint.

The performance results were surprising as none of the four groups had a clear advantage over the others (Figure 3). Pairs with a card did perform slightly better, but this difference did not reach significance. However, it was clear that the more "collective" the system, the fewer extreme results were achieved: i.e. the results were 1 out of 3 or 2 out of 3 wines recognized, rather than 0 out of 3 or 3 out of 3 , which seems to be a general tendency for collective systems.

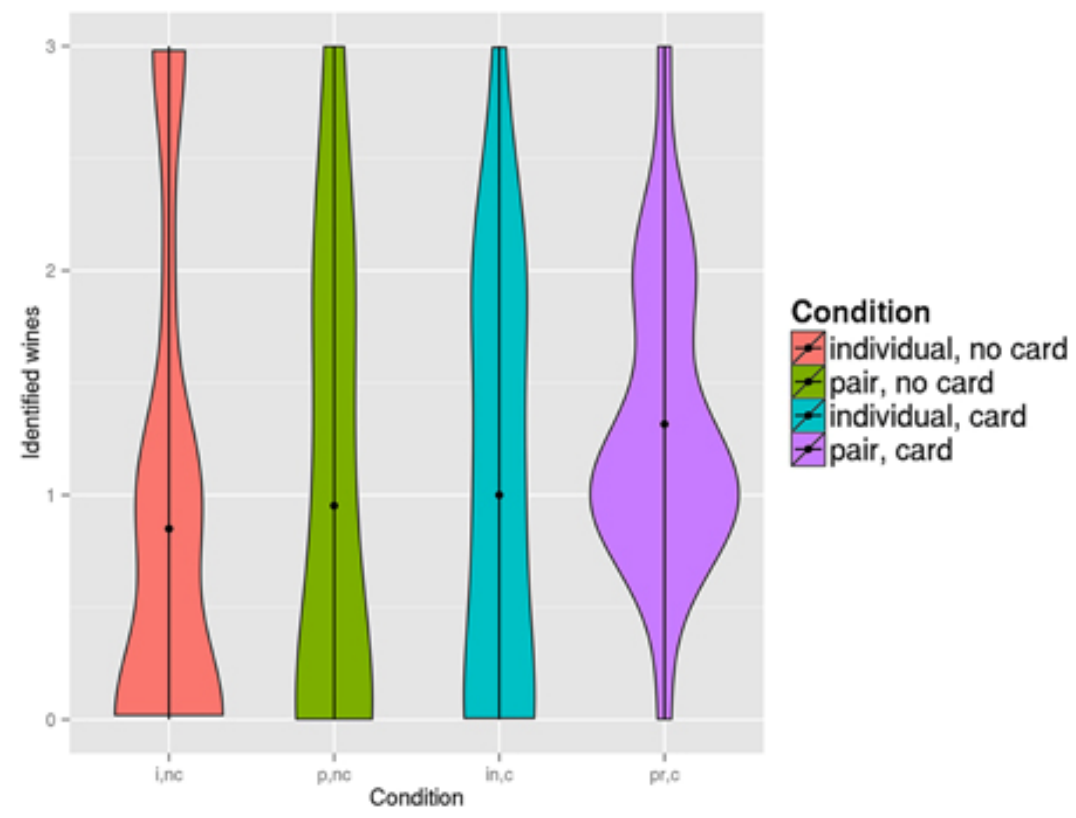

Figure 3 
When it comes to the kinds of errors that were made by the collective systems, the story was much more interesting: applying bias-variance analysis (Domingos, 2000; Gigerenzer \& Brighton, 2009) ${ }^{37}$ to individual wine decisions and characterizing such performance for each system, we found that pairs that could converse spontaneously increased rather than reduced their variance. Variance decreased only in the pair condition with a sommelier card and both the variance and the overall error were significantly smaller than in other conditions. The use of the card did not help the individuals: their biasvariance decomposition and the overall error did not differ.

Since we had evidence that cards were helpful only for reducing variance in pairs, we wanted to see if the quality of the cards prepared by pairs and individuals differed. We treated each card (which had 21 dimensions in which wine had to be characterized) as a point in 21-dimensional space, ranknormalizing the dimensions beforehand. We used linear regression and a silhouette score (a simple measure of cohesion and separation of clusters) to assess the coherence and discriminativeness of each wine description prepared by individuals and pairs. Indeed, the classifications of descriptions obtained by pairs were more coherent and discriminative than those obtained by individuals. They were also more accurate for real pairs than for false pairs (obtained by averaging the randomly paired individual descriptions), which indicates that the constraints brought by another person into the description situation are beneficial and are a result of more complex integration of knowledge through communication than simple averaging.

Analyses of verbal interactions may clarify why the variance did not decrease in the case of pairs communicating spontaneously. Perhaps, we suspected, in this situation which was novel for the pairs, they did not succeed in developing the vocabulary important for selecting helpful dimensions (which was shown to be crucial by Fusaroli et al., 2012), but were still in the "scouting" phase of generating descriptions, rather than pruning and selecting helpful terms as controls in interaction. If this was the case, then we should see a relationship between success in establishing a common vocabulary and performance, especially in the pairs without cards. We took two ratios as a measure of development of shared vocabulary: the proportion of wine descriptors that overlapped in a dyad (common vocabulary) and the proportion of wine descriptors that overlapped between the phases of experiment (how stable the

\footnotetext{
${ }^{37}$ A bias-variance analysis allows for assessing if a classifying system is too rigid in its choices (large, consistent biases, low number of degrees of freedom) or too much influences by each sample (less biases but less ability to generalize, i.e., high variance and hence number of degrees of freedom). Usually a system that is both accurate and flexible will optimize both measures in a kind of tradeoff.
} 
vocabulary is in a given pair). Both measures correlated significantly ( $\mathrm{r}=0.35$ and $r=0.40$ ) with the performance of pairs and, interestingly, did so only for pairs without the sommelier card, thus supporting our hypothesis.

Summarizing, we treated the individual and dyadic systems as complex systems under a variety of influences which are irreducible to a clear-cut set of variables, yet we were able to generate and verify hypotheses about how linguistic controls would influence the degrees of freedom of the systems in specific task situations. Congruently with the interaction-dominant approach to cognition, the complexity and multiscalarity of the phenomena under study does not leave one helpless, but rather forces researchers to look for measures and characterizations of a cognitive system other than the purported componential contributions of hidden theoretical constructs to its performance.

Following the theory of the role of symbols in cognitive systems, in both studies we conceptualized language as a set of constraints that affects the properties of a system: in these cases, a dyadic system. Such a framework emphasizes the existing dynamics and tries to capture ways in which they are harnessed by linguistic controls. It is also very important that in both research endeavors we try to find patterns predicted by the theory in messy, naturalistic data, preserving the ecological validity of the situations without overly constraining them by experimental setups. Nevertheless, even in such messy, naturalistic data, we see clear patterns emerging that can be captured by the dynamical methods we employ. The key is to project the intertwined complex of multi-timescale variables that could have been affected by various forms of linguistic constraints to measures that, according to our theory, would be expected to index the relevant properties of the relevant systems.

\section{Reconciled with complexity}

Recognizing that answers to the most vital questions in the study of cognition cannot involve just few simple factors, a researcher has to reconcile with complexity. In the above, I showed that the dynamical systems approach, amended with a theory of how symbolic structures might be sustained by the dynamics of a system and function as controls on these dynamics, allows this complexity to be embraced and navigated for specific research goals. This approach helps in looking at old problems in a novel way, which generates new questions and predictions concerning novel measures (coupling, stability, error decomposition, dimensionality). The framework provides methods of data analyses and inference schemas for drawing conclusions from the data, thus complementing the traditional methodology and fruitfully linking to it. It does not preclude that under specific conditions the constraints on a systems' performance are strong and stable enough for a componential-like view to be 
used fruitfully to build an approximate theory of functioning and predict the behavior of a system.

The approach comes with a particular research strategy which, in a sense, seems to be more respectful to the nature of the systems under study. The beginning of any research involves careful observation and probing of the system under study, because recognition of the multiplicity of systems and timescales makes one less hasty in deciding on the "proper" system and timescale. Once the decision of bracketing has been made (if it has to be made), it is a "soft bracketing" rather than a cutoff, with a constant preparedness to include larger/smaller systems and/or slower/faster timescales. This observation and probing phase should also result in identification of the regions of functional stability (their importance being judged by social and ethical factors) and finally, investigation of the parameters that stabilize and destabilize these organizations. This is the way to learn how a system works as well as find the 'points of leverage' where a small change delivered at a specific time and place may lead to large effects. The effects are the value-constrained, societal goals, and the realization that interventions lead to systemic reorganization rather than changing isolated components makes one more aware of their consequences. Importantly, no level or system is privileged as a potential site of such points of leverage. For a specific behavior, they can be found on the levels of genes, the nervous or hormonal system, factors in development, learning \& education, and environment structure (physical and social niche) alike. The choice of which ones to use is obviously cost-based; however, more importantly, it is also ethics and value-based.

Finally, approaches which accept complexity provide tools to integrate not only constraints residing in multiple timescales and systems, but also those that are very different in nature: more stable, solidified in physical forms of the body; more flexible, residing in the relationships among forms; and more fleeting, such as situational or task constraints. Some of the constraints will seem constant or ahistorical; others will depend on a particular history of selective events involving the constraining form within a particular system. This property of the approach opens it to many domains of science and provides a common conceptual system which at some level of abstraction connects exact, social and human scientists. The recent upsurge in research within this framework fuels new hope, if not for a von Bertalanffy-like unity of sciences (von Bertalanffy, 1968), then at least for better recognition of the relations among the sciences and specific reasons for limits of predictability in each of them. 


\section{References}

Beim Graben, P., Pinotsis, D., Saddy, D., Potthast, R. 2008. Language processing with dynamic fields. Cognitive Neurodynamics, 2(2): 79-88. http://doi.org/10.1007/s11571008-9042-4

Case, P., Tuller, B. 1995. Individual differences and the acquisition of new phonetic categories. The Journal of the Acoustical Society of America, 98(2892). https://doi.org/10.1121/1.414311

Deary, I.J., Caryl, P.G., Gibson, G.J. 1993. Nonstationarity and the Measurement of Psychophysical Response in a Visual Inspection-Time Task. Perception, 22(10): 12451256. https://doi.org/10.1068/p221245

Domingos, P. 2000. A Unified Bias-Variance Decomposition for Zero-One and Squared Loss.

Fusaroli, R., Bahrami, B., Olsen, K., Roepstorff, A., Rees, G., Frith, C., Tylen, K. 2012. Coming to Terms: Quantifying the Benefits of Linguistic Coordination. Psychological Science, 23(8): 931-939. https://doi.org/10.1177/0956797612436816

Gigerenzer, G., Brighton, H. 2009. Homo Heuristicus: Why Biased Minds Make Better Inferences. Topics in Cognitive Science, 1(1): 107-143. https://doi.org/10.1111/j.17568765.2008.01006.x

Guastello, S.J., Gregson, R.A.M. 2016. Nonlinear Dynamical Systems Analysis for the Behavioral Sciences Using Real Data. CRC Press.

Haken, H., Kelso, J.A.S., Bunz, H. 1985. A theoretical model of phase transitions in human hand movements. Biological Cybernetics, 51(5): 347-356. https://doi.org/10.1007/BF00336922

Healey, P.G.T., Purver, M., Howes, C. 2014. Divergence in Dialogue. PLoS ONE, 9(6): e98598. doi:10.1371/journal.pone.0098598

Heft, H. 1989. Affordances and the Body: An Intentional Analysis of Gibson's Ecological Approach to Visual Perception. Journal for the Theory of Social Behaviour, 19(1): 130. https://doi.org/10.1111/j.1468-5914.1989.tb00133.x

Hock, H.S., Schöner, G., Giese, M. 2003. The dynamical foundations of mo-tion pattern formation: Stability, selective adaptation, and perceptual continuity. Perception \& Psychophysics, 65: 429-457.

Jeka, J.J., Kelso, J.A.S. 1989. The Dynamic Pattern Approach to Coordinated Behavior: A Tutorial Review. ResearchGate, 61. https://doi.org/10.1016/S0166-4115(08)60017-5

Jirsa, V.K., Fuchs, A., Kelso, J.A.S. 1998. Connecting Cortical and Behavioral Dynamics: Bimanual Coordination. Neural Computation, 10(8): 2019-2045. https://doi.org/10.1162/089976698300016954

Kelso, J.A.S. 1995. Dynamic Patterns: The Self-Organization of Brain and Behavior. Cambridge, Mass.: A Bradford Book. 
Leonardi, G., Nomikou, I., Rohlfing, K.J., Rączaszek-Leonardi, J. 2016. Vocal interactions at the dawn of communication: The emergence of mutuality and complementarity in mother-infant interaction. Proceedings of the IEEE ICDL-EpiRob, Cergy-Pontoise.

Merleau-Ponty, M. 1962. Phenomenology of Perception. Routledge.

Nomikou, I., Leonardi, G., Rohlfing, K., Rączaszek-Leonardi, J. 2016. Constructing interaction: the development of gaze dynamics. Infant and Child Development, 25(3): 277295, DOI: 10.1002/icd.1975, (IF=1,14), MNiSW 25.

Orsucci, F., Giuliani, A., Webber, C. Jr., Zbilut, J., Fonagy, P., Mazza, M. 2006. Combinatorics and synchronization in natural semiotics, Physica A: Statistical Mechanics and its Applications, 361(2): 665-676.

Pattee, H.H., Rączaszek-Leonardi, J. 2012. LAWS, LANGUAGE and LIFE (Vol. 7). Dordrecht: Springer Netherlands. Retrieved from http://link.springer.com/10.1007/97894-007-5161-3

Rączaszek, J., Tuller, B., Shapiro, L.P., Case, P., Kelso, S. 1999. Categorization of ambiguous sentences as a function of a changing prosodic parameter: a dynamical approach. Journal of Psycholinguistic Research, 28(4): 367-393.

Rączaszek-Leonardi, J. (in press). How does a word become a message? An illustration on a developmental time-scale. New Ideas in Psychology.

Rączaszek-Leonardi, J. 2010. Multiple Time-Scales of Language Dynamics: An Example From Psycholinguistics. Ecological Psychology, 22(4): 269-285. https://doi.org/10.1080/10407413.2010.517111

Rączaszek-Leonardi, J. 2014. Multiple Systems and Multiple Time Scales of Language Dynamics: Coping with Complexity. Cybernetics \& Human Knowing, 21(1-2): 37-52.

Rączaszek-Leonardi, J. 2016. How does a word become a message? An illustration on a developmental time-scale. New Ideas in Psychology, 42: 46-55. doi:10.1016/j.newideapsych.2015.08.001, (IF=1.44), MNiSW 25

Rączaszek-Leonardi, J., Kelso, J.A.S. 2008. Reconciling symbolic and dynamic aspects of language. New Ideas in Psychology, 26(2): 193-207. https://doi.org/10.1016/j.newideapsych.2007.07.003

Rączaszek-Leonardi, J., Nomikou, I. 2015. Beyond mechanistic interaction: Value-based constraints on meaning in language. Frontiers in Psychology, 6:1579. doi: 10.3389/fpsyg.2015.01579. (IF=2.6)

Rączaszek-Leonardi, J., Nomikou, I., Rohlfing, K.J. 2013. Young Children’s Dialogical Actions: The Beginnings of Purposeful Intersubjectivity. IEEE Transactions on Autonomous Mental Development, 5(3): 210-221. https://doi.org/10.1109/TAMD.2013.2273258

Romero, V., Kallen, R., Riley, M.A., Richardson, M. J. 2015. Can Discrete Joint Action Be Synergistic? Studying the Stabilization of Interpersonal Hand Coordination. Journal of Experimental Psychology. Human Perception and Performance, 41(5): 1223-1235. https://doi.org/10.1037/xhp0000083 
Scholz, J. P., Schöner, G. 1999. The uncontrolled manifold concept: identifying control variables for a functional task. Experimental Brain Research, 126(3): 289-306. https://doi.org/10.1007/s002210050738

Schöner, G., Kelso, J.A. 1988. Dynamic pattern generation in behavioral and neural systems. Science (New York, N.Y.), 239(4847): 1513-1520.

Thelen, E. 1992. Development as a Dynamic System. Current Directions in Psychological Science, 1(6): 189-193.

Thelen, E., Smith, L.B. 1996. A Dynamic Systems Approach to the Development of Cognition and Action (Reprint edition). Cambridge, Mass.: A Bradford Book.

Tuller, B., Case, P., Ding, M., Kelso, J.A. 1994. The nonlinear dynamics of speech categorization. Journal of Experimental Psychology. Human Perception and Performance, 20(1): 3-16.

Van Orden, G.C., Holden, J. G., Turvey, M.T. 2003. Self-organization of cognitive performance. Journal of Experimental Psychology. General, 132(3): 331-350. https://doi.org/10.1037/0096-3445.132.3.331

Von Bertalanffy, L. 1968. General Systems Theory. New York: George Braziller.

Wallot, S., Hollis, G., Rooij, M. van. 2013. Connected Text Reading and Differences in Text Reading Fluency in Adult Readers. PLOS ONE, 8(8). e71914. https://doi.org/10.1371/journal.pone.0071914

Wallot, S., Van Orden, G.C. 2011a. Grounding Language Performance in the Anticipatory Dynamics of the Body. Ecological Psychology, 23(3): 157-184. https://doi.org/10.1080/10407413.2011.591262

Wallot, S., Van Orden, G.C. 2011b. Nonlinear analyses of self-paced reading. The Mental Lexicon, 6(2): 245-274. https://doi.org/10.1075/ml.6.2.02wal

Zanone, P.G., Kelso, J.A. 1997. Coordination dynamics of learning and transfer: collective and component levels. Journal of Experimental Psychology. Human Perception and Performance, 23(5): 1454-1480.

Zubek, J., Denkiewicz, M., Dębska, A., Radkowska, A., Komorowska-Mach, J., Litwin, P., ... Rączaszek-Leonardi, J. 2016. Performance of Language-Coordinated Collective Systems: A Study of Wine Recognition and Description. Frontiers in Psychology, 7. https://doi.org/10.3389/fpsyg.2016.01321 